\title{
Study on applicability of RFT to traveling analysis of wheel with grousers: Comparison with DEM analysis as a virtual test
}

\author{
Hirotaka Suzuki, Kota Katsushima, Shingo Ozaki* \\ Division of System Research, Faculty of Engineering, Yokohama National University, Tokiwadai 79-5, Hodogaya-ku, Yokohama 240-8501, Japan
}

\section{A R T I C L E I N F O}

Article history:

Received 8 March 2018

Accepted 9 January 2019

\section{Keywords:}

RFT

Wheel-soil interaction

Grouser

Trafficability

Slippage

\begin{abstract}
A B S T R A C T
We studied a method to calculate the traveling characteristics of small and lightweight rovers at low cost. Specifically, discrete element method (DEM) was considered as a high-cost and high-accuracy virtual test, and plate tests and traveling analyses of the wheel with grousers were performed. Meanwhile, we adopted resistive force theory (RFT) as a low-cost analysis method and confirmed its applicability to the traveling of wheels with grousers by comparing it with the results obtained by DEM analysis of a loose frictional soil. First, we determined the scale factor necessary for RFT calculation by DEM analysis of the plate penetration test. Then, DEM wheel models with three types of grouser were prepared, and we compared the drawbar-pull under several levels of slippage with the results obtained by RFT. Although RFT cannot sufficiently consider the influences of the shearing of the soil and of distance between grousers, it found that the effects of grousers can be examined at low cost by RFT.
\end{abstract}

(c) 2019 ISTVS. Published by Elsevier Ltd. All rights reserved.

\section{Introduction}

In future moon and planetary exploration activities, various operations are expected on soft soils, such as the landing of landers, drilling and sampling of the ground, installation of observation equipment, and construction of bases. To realize these tasks, rovers and robots moving on soft ground are indispensable, and related research has been conducted.

However, it is widely known that the surface of the moon/ planet is covered with fine sand, called the "regolith," under a microgravity environment. Therefore, vehicles can easily sink and become stuck (Heiken et al., 1991; Kobayashi et al., 2009; Johnson and King, 2010; Wong, 2012). In addition, there are various sand ripples on the ground of Mars, and these are also known to interfere with wheel traveling (Zhou et al., 2014; Arvidson et al., 2017). To proceed with tasks while avoiding getting stuck, it is effective to establish a traveling plan by simulators based on the terramechanics analysis method, which can accurately and quickly evaluate the traveling characteristics. There are various approaches to calculating the traveling characteristics of off-the-road vehicles on soft ground. Representative examples include numerical analysis methods, such as discrete element method (DEM) (Nakashima et al., 2007, 2010; Li et al., 2010; Knuth et al., 2012; Smith and Peng, 2013; Jiang et al., 2014; Johnson et al., 2015; Nishiyama

\footnotetext{
* Corresponding author.

E-mail address: s-ozaki@ynu.ac.jp (S. Ozaki).
}

et al., 2016) and finite element method (FEM) (Fervers, 2004, Chiroux et al., 2005; Hambleton and Drescher, 2008, 2009; Xia, 2011; Ozaki et al., 2015; Ozaki and Kondo, 2016), focusing on the interaction between the traveling part and the soil. In these methods, advanced contact models and constitutive models are introduced, and thus, the soil deformation and movement can be accurately analyzed by adopting fine discretization (fine particles or elements). However, it is difficult to apply DEM and FEM to the analysis of the behavior of the entire vehicle because high calculation cost is required.

Meanwhile, it is possible to evaluate the interaction between soil and machine within the framework of the multibody dynamics using common engineering terramechanics models, such as the Wong and Reece model (based on Bekker's work). This type of semi-empirical approach can be applied to the behavior analysis of the entire vehicle at a relatively low cost (Ishigami et al., 2007; Sutoh et al., 2012; Zhou et al., 2014). Furthermore, in general, although the rover's wheels are accompanied by grousers or lugs to improve the traveling performance, this semi-empirical approach can also be applied to such cases. For instance, Sutoh et al. (2011) analyzed wheels having grousers by combining the theory of earth pressure with the Wong and Reece model. However, there are restrictions on calculable shapes, and problems, such as being unsuitable for arbitrary traveling parts, remain.

On the other hand, resistive force theory (RFT) has been proposed to empirically estimate the reaction force of a rigid body moving inside a loose granular medium. Although the RFT was 
originally developed for viscous drag problems, RFT has attracted renewed attention in recent years as a method that can easily reflect the interaction between a granular medium and a traveling part having arbitrary shapes (including parts, such as legs, interacting with soil) (Li et al., 2013; Zhang and Goldman, 2014; Askari and Kamrin, 2016; Suzuki et al., 2016; Slonaker et al., 2017; Suzuki and Ozaki, 2017). The RFT has been applied to multibody dynamics analysis for legged mobile robots (Li et al., 2013; Zhang and Goldman, 2014) and wheel traveling analysis (Suzuki et al., 2016; Slonaker et al., 2017; Suzuki and Ozaki, 2017), and its applicability for terramechanics problems has been verified. Furthermore, Slonaker et al. (2017) utilizes the intrusion force evaluated by RFT to derive general scaling relations for locomotion in granular media. Note that Askari and Kamrin (2016) have already explained the physical reasons why granular media having complex behaviors locally can be expressed by RFT. According to their research, RFT may be applicable to cohesive media unless a target soil is a velocity-dependent viscous medium. In addition, they are organizing a new family of resistive-force-obeying material, and it has been shown that RFT shows good prediction accuracy with loose granular media. Therefore, if this theory can also be applied to traveling analysis of wheels with grousers (or lugs), RFT is expected to be an effective method in the field of terramechanics.

In this study, we examine the effectiveness of RFT on the analysis of wheels having grousers by comparing RFT results with DEM analysis results, because DEM is already recognized as an effective numerical analysis method in the field of terramechanics. The strategy of this research is as follows:

1. DEM can demonstrate high analytical accuracy if appropriate conditions are set for the interaction between soil and machine (Nakashima et al., 2007, 2010; Li et al., 2010; Knuth et al., 2012; Smith and Peng, 2013; Jiang et al., 2014; Johnson et al., 2015; Nishiyama et al.,2016).

2. We conducted wheel traveling analyses for a loose frictional soil as a virtual test based on the DEM. We used rigid wheel models with several patterns of grousers.

3. Using a DEM ground model (testbed) with the same condition of wheel traveling analysis, we conducted a plate intrusion test to obtain parameters for RFT analysis.

4. Using the parameters obtained in Step 3, we conducted an RFTbased wheel traveling analysis under the same conditions as in Step 2.

5. If the results of the DEM can be satisfactorily reproduced by RFT, it will lead to the realization of multibody dynamics analysis with high accuracy and low cost for complex wheel shapes. Also, if there is some discrepancy between them, it is possible to clarify the cause and to consider the scope of application.

We briefly review of the outline of RFT in Section 2. In Section 3, we conduct plate tests using both DEM and RFT and compare these results. We then identify the parameters for calculation of RFT. In Section 4, we calculate the traveling characteristics of the wheels with grousers using the obtained RFT parameters, and we compare them with the calculation results using DEM. Furthermore, we also discuss the validity of RFT and its limitations. Finally, in Section 5, we present our conclusions.

\section{Resistive force theory}

RFT is a model utilizing a set of hypotheses about local drag forces to approximate resistance on general solid surfaces moving in granular soils near the surface. It has been shown that RFT can be applied to granular media (Li et al., 2013; Zhang and Goldman, 2014; Askari and Kamrin, 2016; Suzuki et al., 2016; Slonaker et al., 2017; Suzuki and Ozaki, 2017).
In short, RFT is a method that can reflect the posture and motion direction of the plate in the calculation of the reaction force occurring on a small plate in granular media. The intrusion/extrusion stress on the plate at depth $z$ from the ground surface is given as follows:

$$
\left.\begin{array}{l}
\sigma_{x}=\alpha_{x} z \\
\sigma_{z}=\alpha_{z} z
\end{array}\right\}
$$

where $\sigma_{x}$ and $\sigma_{z}$ are the horizontal and vertical intrusion/extrusion stresses, respectively; $\alpha_{x}$ and $\alpha_{z}$ are the stiffness in each direction, and they are given as functions of the orientation angle $\beta$ and velocity vector angle $\gamma$ of the plate, defined in Fig. 1.

An important part of the RFT is the choice of functions $\alpha$. In this study, the following equations are adopted from Li et al. (2013), although the stiffness $\alpha$ is generally obtained by the plate test in which various postures (orientation angles) and motion directions (velocity vector angles) of the small plate are set.

$$
\left.\begin{array}{l}
\alpha_{x}(\beta, \gamma)=\zeta \sum_{m=-1}^{1} \sum_{n=0}^{1}\left[C_{m, n} \cos 2 \pi\left(\frac{m \beta}{\pi}+\frac{n \gamma}{2 \pi}\right)+D_{m, n} \sin 2 \pi\left(\frac{m \beta}{\pi}+\frac{n \gamma}{2 \pi}\right)\right] \\
\alpha_{z}(\beta, \gamma)=\zeta \sum_{m=-1}^{1} \sum_{n=0}^{1}\left[A_{m, n} \cos 2 \pi\left(\frac{m \beta}{\pi}+\frac{n \gamma}{2 \pi}\right)+B_{m, n} \sin 2 \pi\left(\frac{m \beta}{\pi}+\frac{n \gamma}{2 \pi}\right)\right]
\end{array}\right\} .
$$

Eq. (2) was obtained by applying the approximation based on the Fourier transform to the results obtained by the plate test. In this study, the coefficients $A, B, C$, and $D$ used for the Fourier transform are cited from Li et al. (2013), and the values are shown in Table $1 . \zeta$ is called the scale factor (grain structure coefficient), and it depends on the grains, intruder roughness, and value of gravity. As shown by Eq. (3), the scale factor $\zeta$ is simply determined from the stiffness of the vertical plate intrusion test, i.e.,

$\zeta \approx \alpha_{z}\left(0, \frac{\pi}{2}\right)$

Fig. 2 shows the distribution of stiffness $\alpha$ with respect to the orientation angle $\beta$ and the velocity vector angle $\gamma$ when the values shown in Table 1 are used. Here, the scale factor $\zeta$ is 1.0. In the RFT, because the distribution characteristic of $\alpha$ shown in Fig. 2 does not depend on the type and state of the granular media, the parameter used for analysis is only $\zeta$. For this reason, we can determine $\zeta$ by comparing it with the result using the DEM under the condition of Eq. (3) (see Section 3).

The stress applying in each part can be obtained by multiplying $\alpha$ to sinkage $z$, as shown in Eq. (1). Therefore, by discretizing the shape of the traveling part with small plates and by assuming a simple superposition principle, we can calculate the entire force in each direction.

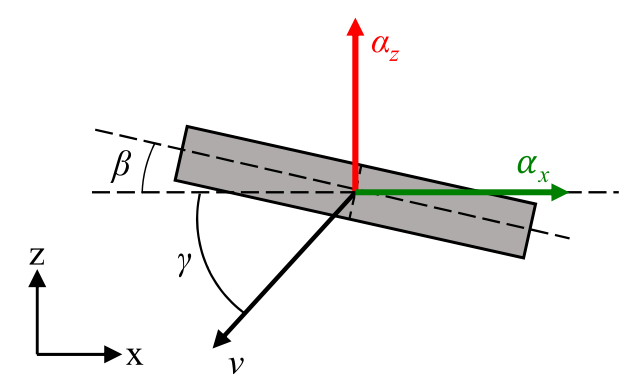

Fig. 1. Definition of the orientation angle $\beta$, velocity vector angle $\gamma$, and stresses $\sigma_{x}$ and $\sigma_{z}$. 
Table 1

Coefficients for RFT (data after Li et al., 2013).

\begin{tabular}{|c|c|c|c|c|c|c|c|c|}
\hline$A_{0,0}$ & $A_{1,0}$ & $B_{1,1}$ & $B_{0,1}$ & $B_{-1,1}$ & $C_{1,1}$ & $C_{0,1}$ & $C_{-1,1}$ & $D_{1,0}$ \\
\hline 0.206 & 0.169 & 0.212 & 0.358 & 0.055 & -0.124 & 0.253 & 0.007 & 0.088 \\
\hline
\end{tabular}
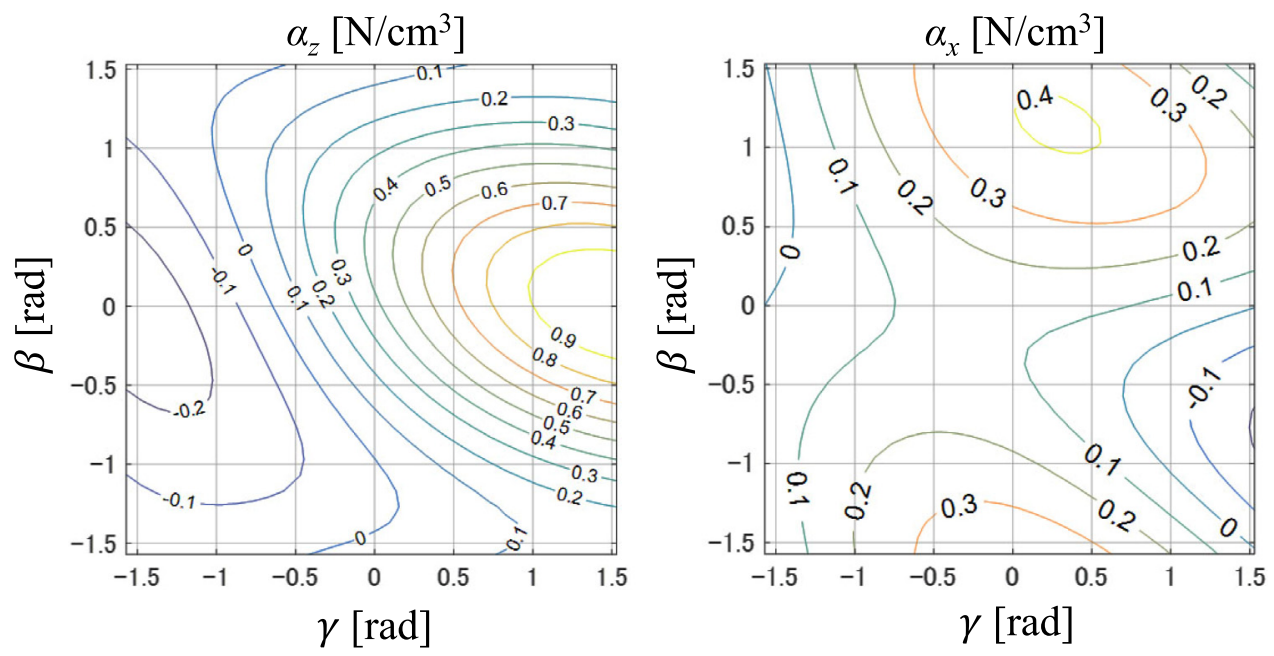

Fig. 2. Distributions of $\alpha_{x}$ and $\alpha_{z}$ obtained by Eq. (1), where $\zeta=1$.

\section{Virtual plate test using DEM}

\subsection{Analysis model}

As a virtual plate intrusion/extrusion test, we conducted DEM analysis. In this study, the discrete element function of the commercial software LS-DYNA was used (LSTC, 2017). Fig. 3 shows the DEM analysis model. Here, the soil particles were filled in the testbed by free falling from the upper side, to realize a loose deposit condition (the density is $905.4 \mathrm{~kg} / \mathrm{m}^{3}$ ). We adopted the Voigt model for the treatment of particle contact. Table 2 shows the parameters used for the DEM analysis. Here, we chose DEM parameters as that the angle of repose corresponds to typical sandy soil.

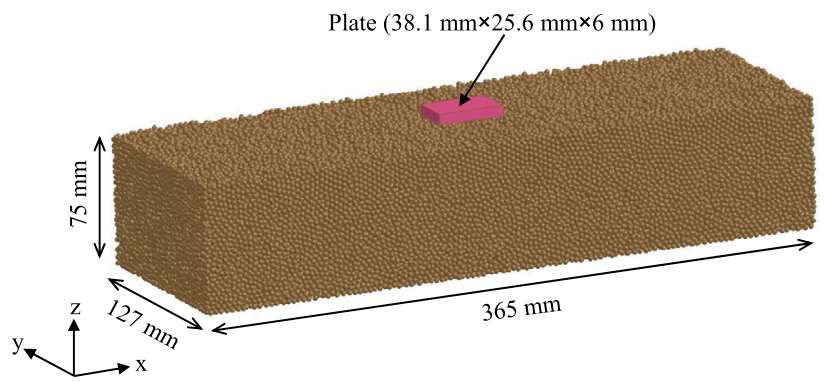

Fig. 3. DEM analysis model for plate tests.

Table 2

Analysis condition of DEM model.

\begin{tabular}{ll}
\hline Particle diameter $[\mathrm{mm}]$ & 3.00 \\
Static friction coefficient & 0.750 \\
Dynamic friction coefficient & 0.750 \\
Density of particle $\left[\mathrm{kg} / \mathrm{m}^{3}\right]$ & 1550 \\
Young's modulus $[\mathrm{MPa}]$ & 100 \\
Poisson ratio & 0.4 \\
\hline
\end{tabular}

The plate was set to be a rigid body, and the prescribed forced displacement was given corresponding to the specified orientation angle $\beta$ and velocity vector angle $\gamma$. Here, the plate was the same size as that used for the real test (Li et al., 2013), and we assumed that the friction coefficient between the soil particle and plate is the same as that between the soil particles.

\subsection{Determination of scale factor $\zeta$}

First, the scale factor was determined using the results of the virtual plate test by DEM. Because the scale factor is obtained from Eq. (3), we analyzed the condition in which the horizontal plate intrudes vertically downward. Fig. 4 shows the stress-sinkage

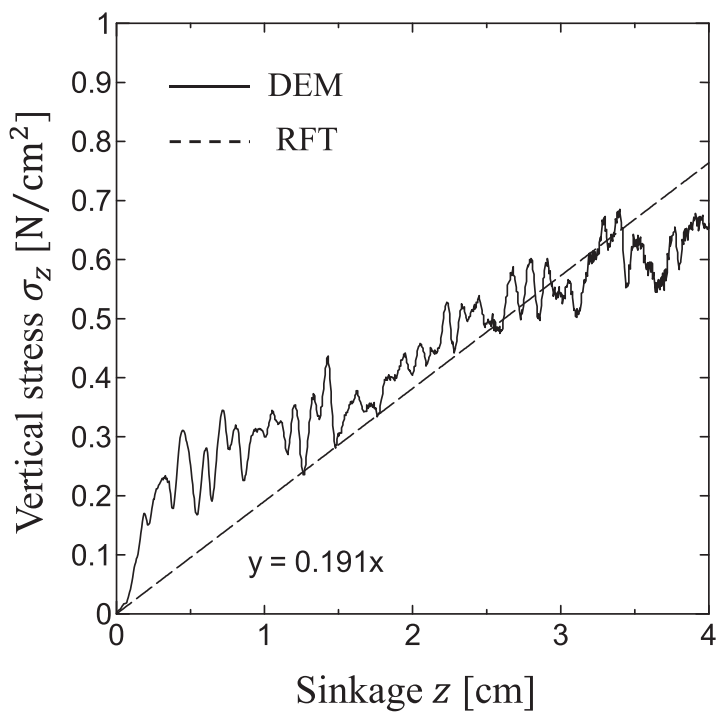

Fig. 4. Comparison of the vertical stress vs. sinkage relation between DEM and RFT. Here, $\beta=0$ and $\gamma=\pi / 2$. The scale factor is determined as $\zeta=0.191$ by the linear approximation. 

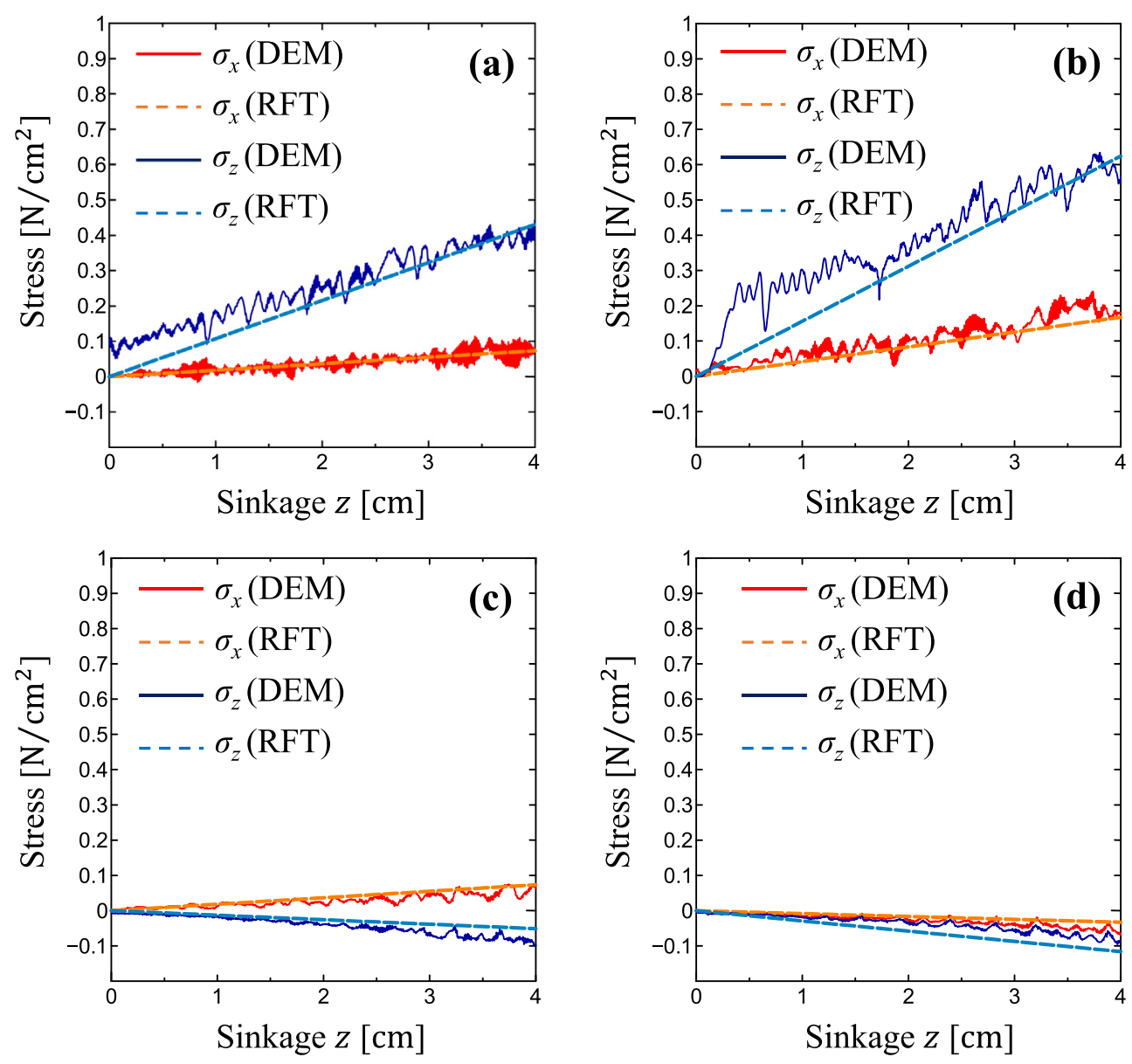

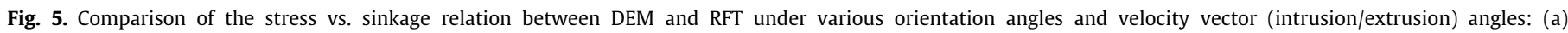
$\beta=0, \gamma=\pi / 4$; (b) $\beta=\pi / 4, \gamma=\pi / 2$; (c) $\beta=0, \gamma=-\pi / 4$; and (d) $\beta=\pi / 4, \gamma=-\pi / 2$.

relationship obtained by DEM and RFT. Here, the solid line is the result of DEM, while the dashed line is the result of RFT. Because $\alpha$ is defined as the stiffness of the stress-sinkage relationship, the scale factor can be determined by the inclination. In general, although the stress-sinkage relationship shows nonlinearity, the RFT result shown in Fig. 4 was approximated to a straight line passing through the origin by the least-squares method. Thus, the scale factor of the soil model shown in Fig. 3 was determined to be 0.191.

Next, to confirm the validity of the obtained scale factor, we compared the stress-sinkage relationships between DEM and RFT under several orientation angles $\beta$ and velocity vector angle $\gamma$. The scale factor of RFT used was $\zeta=0.191$, which was already determined. Fig. 5 shows the stress-sinkage relations under two conditions of intrusion and two conditions of extrusion. The solid line is the result of DEM, while the dashed line is the result of RFT. It is confirmed from Fig. 5 that the stress-sinkage relations in the horizontal and vertical directions obtained by the RFT are in good agreement with those obtained by DEM. The abovementioned results show that the virtual plate test using DEM can be reproduced by RFT.

\section{Traveling analysis of wheel with grousers}

Because the consistency of DEM and RFT was confirmed in the plate test, in this section, we confirm the applicability of RFT to the wheel traveling analysis with more-complex shapes and movements.

\subsection{Analysis model and boundary condition}

Fig. 6 shows the DEM analysis model for the single-wheel traveling analysis, which is used as the virtual test. We adopted three types of wheel, as shown in Table 3, while the condition of the testbed was the same as in Fig. 3. The width of the wheel and that of the testbed were the same; thus, the plane strain condition was assumed.

In the DEM and RFT analyses, the slippage of the wheel was controlled by independently setting the prescribed traveling velocity $v$ and rotation velocity $\omega$. For verification of RFT, we focused on the variation of the coefficient of traction, which is defined by the ratio of the drawbar-pull and wheel load. The slippage $s$ is defined as follows:

$s=1-\frac{v}{D \omega / 2}$

where $D$ is the diameter of wheel (see Fig. 7). In the analyses, the rotation velocity $\omega$ of the wheel was fixed at $0.5 \mathrm{rad} / \mathrm{s}$, while the traveling velocity $v$ was adjusted. In addition, a lightweight and compact vehicle was assumed for these analyses, and the wheel load was set to be $20 \mathrm{~N}$.

Fig. 7 shows the wheel with grousers. The figure also shows the schematic diagram of the orientation angle $\beta$ and intrusion angle $\gamma$ when discretizing the wheel part and grouser part by small plates, to apply the RFT. Here, $r$ is the distance from the center of the wheel to the center of each plate constituting wheel surface and grousers. In the RFT analysis, the drawbar-pull is evaluated by 


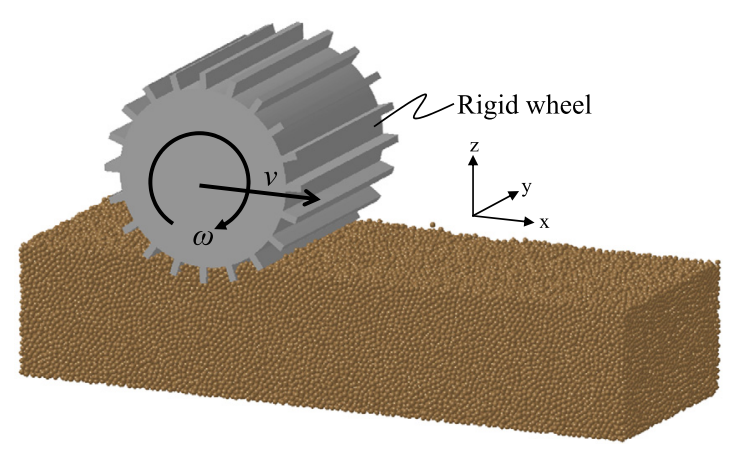

Fig. 6. DEM analysis model for the single wheel traveling test. The ground condition is the same as the model shown in Fig. 3.

Table 3

Specifications of wheels.

\begin{tabular}{llll}
\hline & Wheel A & Wheel B & Wheel C \\
\hline Number of grouser & 20 & 20 & 15 \\
Height of grouser $h_{g}[\mathrm{~mm}]$ & 6 & 9 & 9 \\
Thickness of grouser $t_{\mathrm{g}}[\mathrm{mm}]$ & 4 & & \\
Diameter $D[\mathrm{~mm}]$ & 100 & & \\
Width $b[\mathrm{~mm}]$ & 127 & & \\
\hline
\end{tabular}

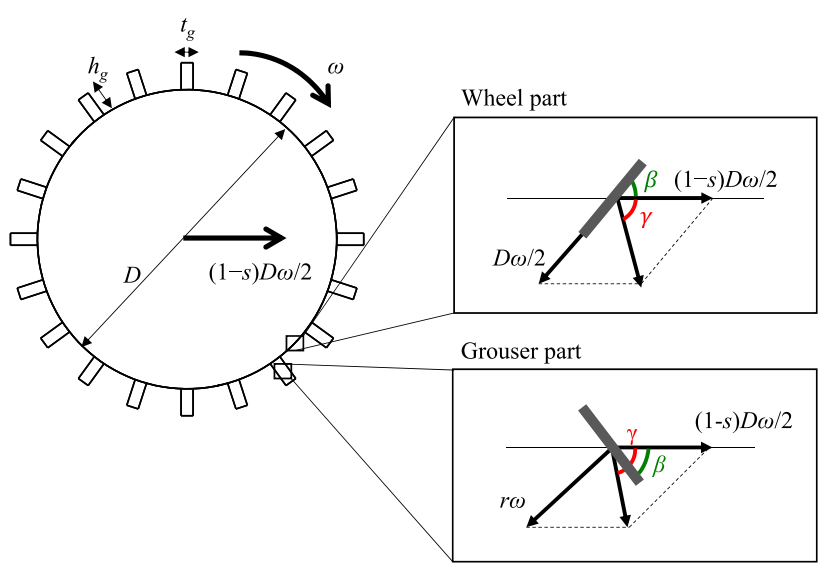

Fig. 7. Application of RFT to wheel with grousers.

superposing the horizontal stress $\sigma_{x}$ on each plate. A convergence calculation of sinkage at each time step must be performed to ensure the equilibrium between the wheel load and the vertical load, which is obtained by superposing the vertical stress $\sigma_{z}$ on each plate. In this study, the wheel surface part was discretized by a $0.5 \mathrm{~mm}$ length of plates. The grouser part was discretized by 30 plates, while wheel part was discretized by 0.01 rad intervals. We also conducted the RFT analysis under the condition that the discretization was further refined, and we confirmed that the influence was small enough.

Eq. (2) is the essence of calculation of RFT, and it is derived by the plate intrusion/extrusion test. Therefore, to apply RFT to a closed shape, such as wheel, ingenuity is required. For instance, in the case where a negative vertical stress is calculated on wheel parts (extrusion case), it corresponds to the force acting on the inside of the wheel. As shown in Fig. 6, however, because there is no granular medium inside the closed-shape wheel, that situation is different from the actual phenomenon. Therefore, in this study, we assumed that no stress is generated on the wheel surface (rear area in the traveling direction) where the vertical stress becomes negative. In addition, the distribution maps of $\alpha_{x}$ and $\alpha_{z}$ shown in
Fig. 2 correspond to range in which $-\pi / 2 \leqslant \beta, \gamma \leqslant \pi / 2$ and the definition of Fig. 1. Therefore, when the velocity vector $\gamma$ at the calculation points is in the positive direction of x-axis, to apply RFT with in the distribution map range, it is necessary to change the calculation method. In this study, when the horizontal component of the velocity vector inverts with respect to the reference definition shown in Fig. 1, the reference plane defining $\beta$ and $\gamma$ was also reversed. Thus, the sign of resistive force calculated by RFT in the horizontal direction was also reversed.

\subsection{Comparison of results obtained by DEM and RFT}

Because the virtual test bed of the DEM analysis model shown in Fig. 6 was the same as that of the plate tests, the same scale factor $\zeta$ already determined was used for comparison of results between by DEM and RFT (see Section 3.1).

Figs. 8(a), 9(a), and 10(a) show variations in the coefficient of traction as a function of elapsed time under each slippage obtained by DEM analysis using wheels A, B, and C. Here, a low-pass filter was used (cutoff frequency is $3 \mathrm{~Hz}$ ) for the results of DEM, as the fluctuation was large. As can be seen from the figure, the drawbar-pull fluctuates owing to the influence of grousers. Furthermore, the drawbar-pull in steady traveling conditions increases with slippage and height of grousers in the range of the examined conditions.

Figs. 8(b), 9(b), and 10(b) show variations in the drawbar-pull as a function of elapsed time under each slippage obtained by RFT analysis using three types of wheel. As with DEM analysis results, the drawbar-pull in steady traveling conditions increases with slippage and the height of grousers in the range of examined conditions. In the RFT, the effect of slippage is reflected by the change in the velocity vector angle $\gamma$. In addition, the periodic fluctuation of the drawbar-pull by grouser is also reflected owing to the change in $\gamma$ and $\beta$ during traveling. Comparing the state of fluctuation between three types of wheel, it can be seen that the amplitude of the drawbar-pull at the same slippage becomes higher together with grouser height. Furthermore, it is also seen from the figures that the period of fluctuation is influenced by the number of grousers. From this fact, it can be confirmed that the RFT makes analysis reflecting the shape of the wheel possible. Furthermore, as has been observed in experiments (e.g., Shikanai et al., 2000), after the starting of traveling under a constant slippage, drawbar-pull increases gradually and then converges to a steady value.

To examine the applicability of RFT in more detail, we created the stress distribution on the wheel surface during steady traveling, as shown in Fig. 11. Here, the stress distribution is the result of Wheel A. The red lines indicate stress generated in the horizontal direction, while the blue lines indicate stress generated in the vertical direction. The length of the lines corresponds to the magnitude of the stresses, and the direction of the lines extending from the wheel surface corresponds to the direction of the stresses applied to the wheel. Compared with the stress in the horizontal direction, the area where the stress acts on the opposite side in the traveling direction (left direction in the figure) at 0.1 slippage is wider than that at 0.5 slippage. Furthermore, the distribution area of stress in the vertical direction becomes large with the increase in slippage. This is because the translational velocity per unit length increases with low slippage, and the region where the velocity vector angle $\gamma$ is inclined forward increases. Thus, the phenomenon that the drawbar-pull increases with slippage is naturally expressed by RFT. A similar tendency was also confirmed in Wheels B and C. However, it should be noted that the relationship between sinkage and slippage cannot be properly expressed by RFT, as will be explained in a later discussion. 

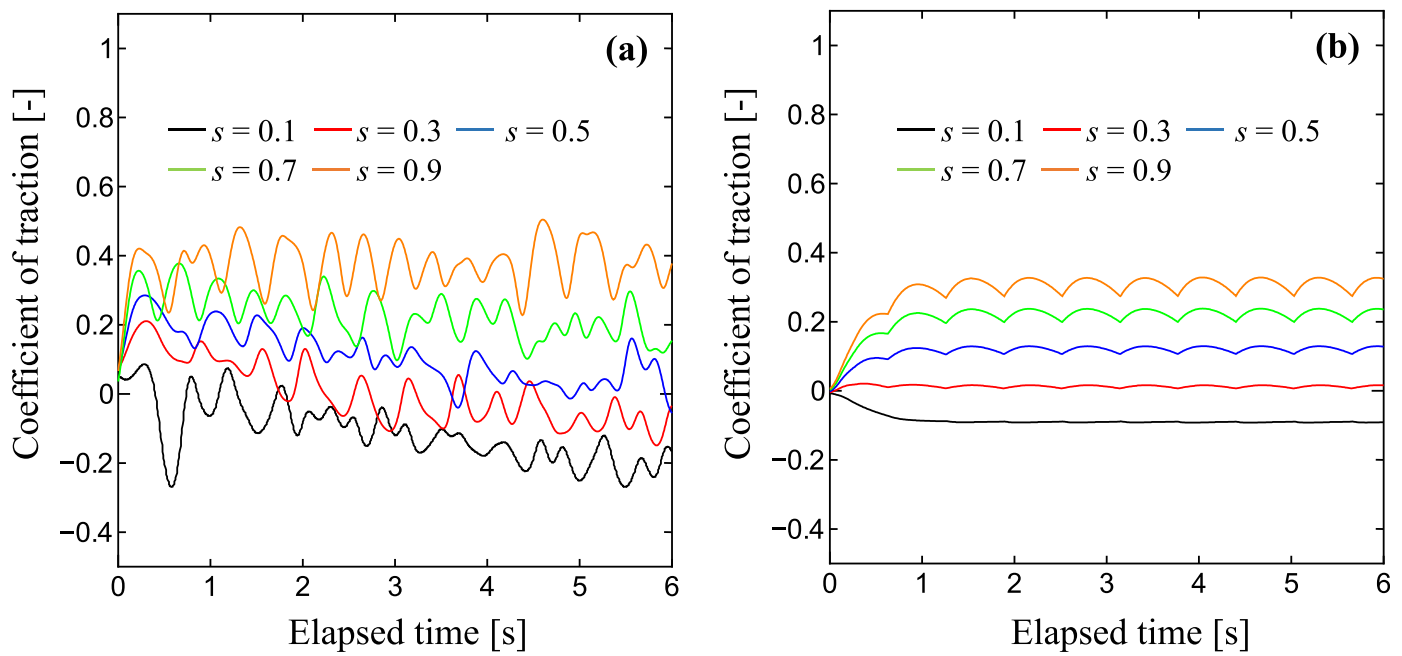

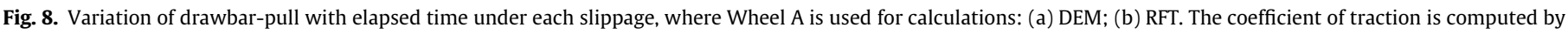
dividing the drawbar-pull by the constant wheel load.
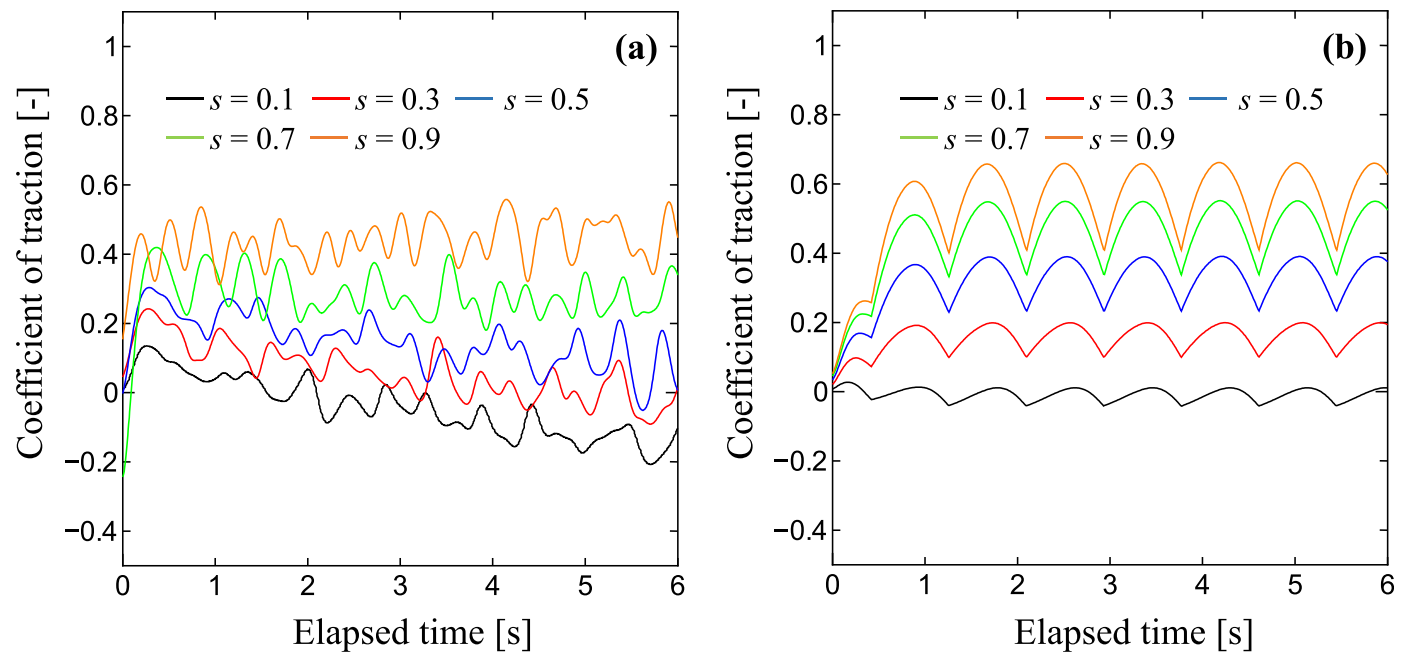

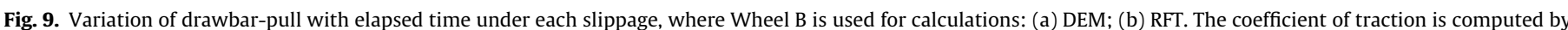
dividing the drawbar-pull by the constant wheel load.
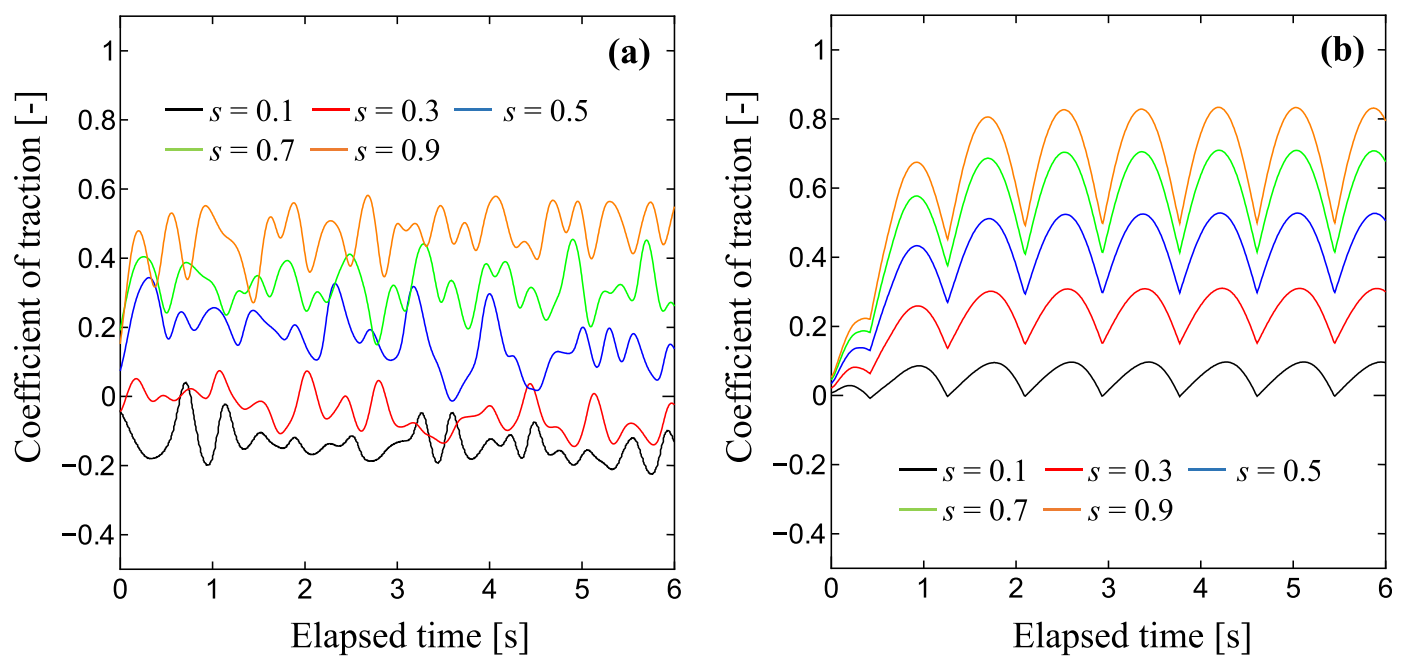

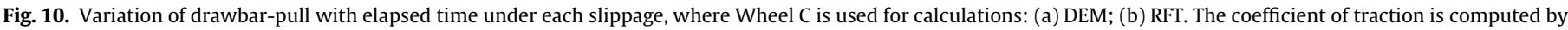
dividing the drawbar-pull by the constant wheel load. 

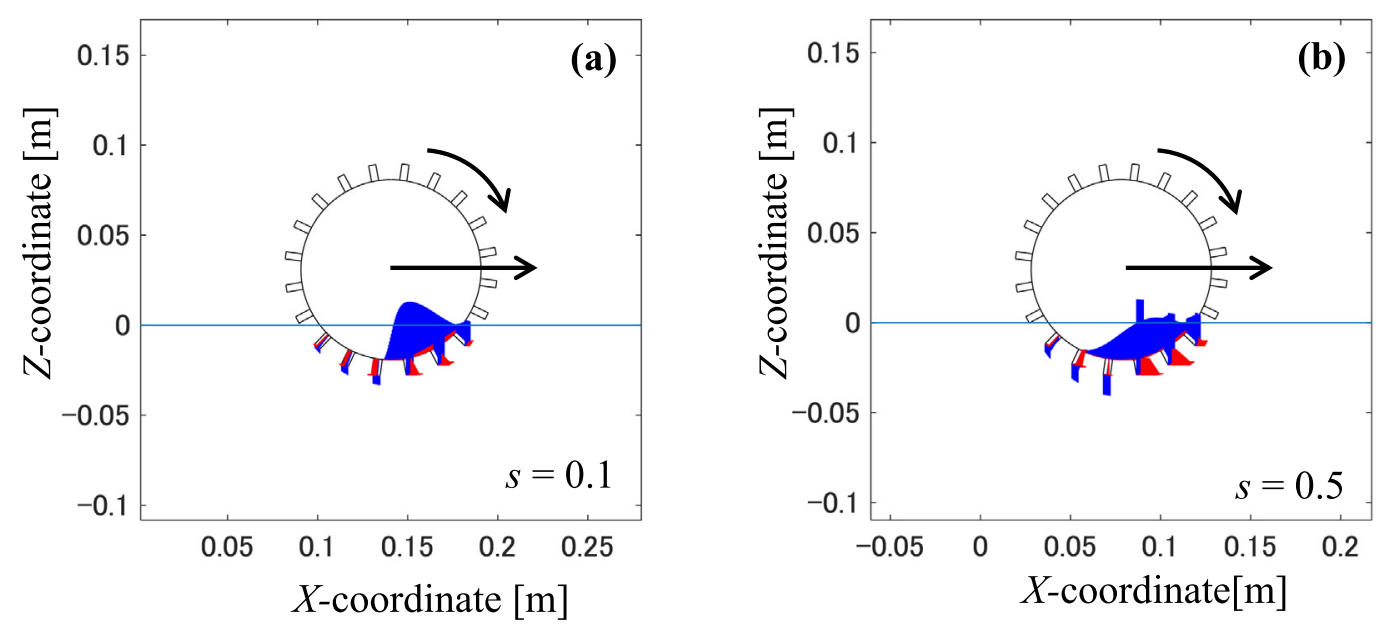

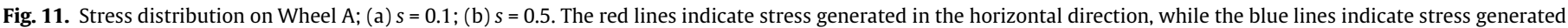
in the vertical direction. (For interpretation of the references to colour in this figure legend, the reader is referred to the web version of this article.)

Fig. 12(a) and (b) show the relationship between the steadystate coefficient of traction and slippage obtained by DEM and RFT, respectively. The DEM results show a similar tendency to that reported in Sutoh et al. (2012). Furthermore, the qualitative tendency obtained by RFT that the drawbar-pull increases with slippage is in reasonably good agreement with DEM results. To confirm the versatility of RFT analysis, we conducted both DEM and RFT analyses using another soil model whose density is higher than that of Figs. 3 and 6. Here, the scale factor $\zeta$ obtained by the virtual penetration test using DEM was 0.524. Fig. 13 shows the relationship between the coefficient of traction in steady state and slippage, where Wheel B was used for the analyses. As can be confirmed from the figure, the traveling characteristics of wheels with grousers can be evaluated at low cost by RFT, even in different soil conditions.

However, there is no quantitative agreement between the DEM and RFT analyses, and the consistency of the influence of grousers (height and number) is not found. The cause of this will be discussed in the next section.

\section{Discussion}

In this section, we discuss the reason why the relationships between the steady-state drawbar-pull and slippage are different

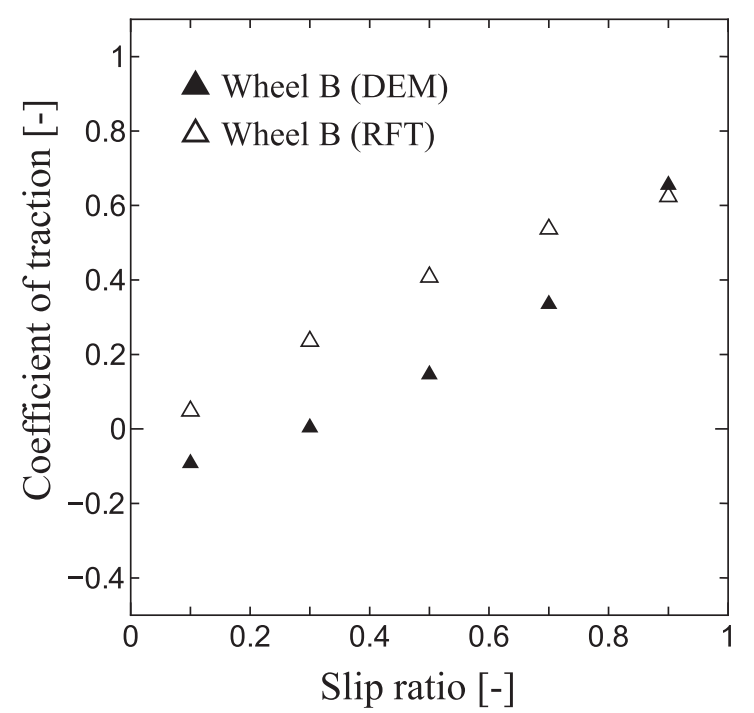

Fig. 13. The relationship between average coefficient of traction in steady state and slippage using Wheel B: (a) DEM; (b) RFT. Here, the different soil condition, which is stiffer than in Figs. 3 and 6, is used for DEM and RFT analyses. The scale factor $\xi$ is 0.524 .
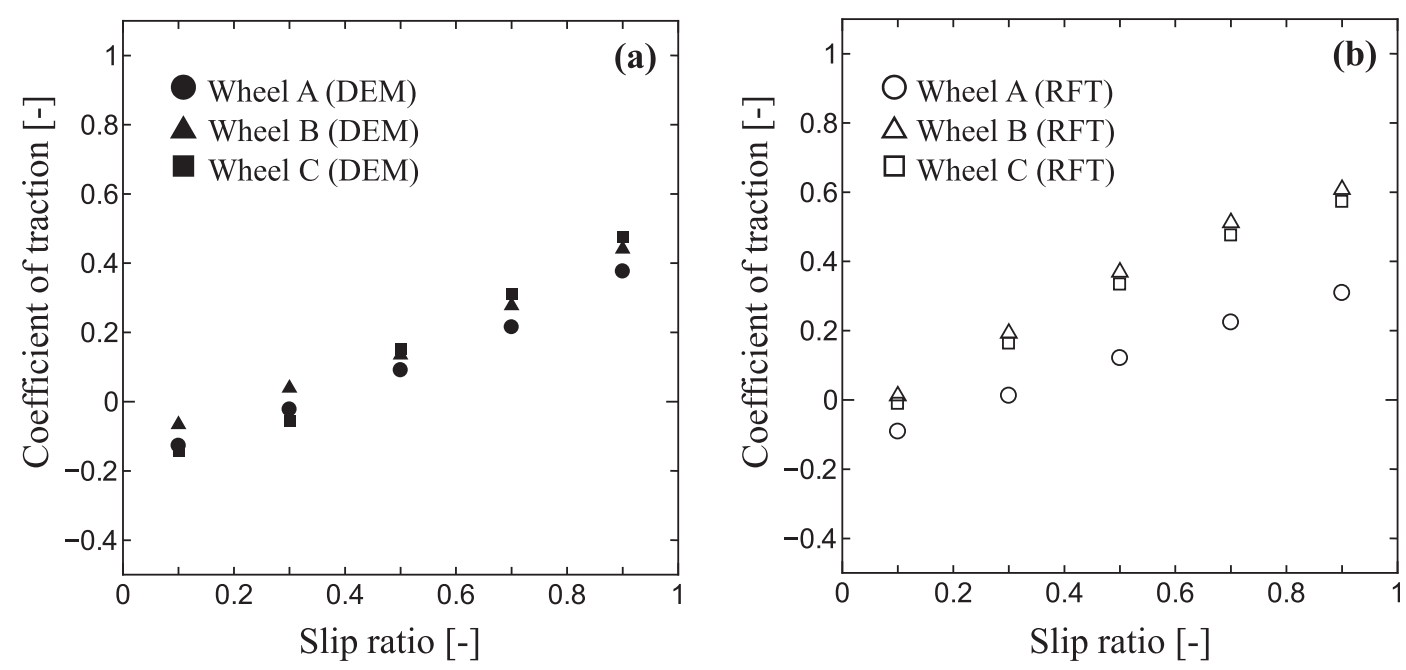

Fig. 12. The relationship between average coefficient of traction in steady state and slippage: (a) DEM; (b) RFT. 
in DEM and RFT. The first reason is that shearing of soil is not considered sufficiently in the calculation of RFT. In general, when the wheel with grousers travels on soft ground, particles are packed between grousers. Hence, an additive driving force is generated by forming a shear plane between soil particles during traveling.

Fig. 14 is a contour map of the absolute value of the particle velocity during wheel traveling obtained by DEM analysis using Wheel B. Here, the slippage is 0.5 . As is confirmed from the figure, the particles between the grousers have large velocity, and the shearing between the particles is expressed in the DEM analysis. In addition, as RFT is targeted for the movement (like a swim) of

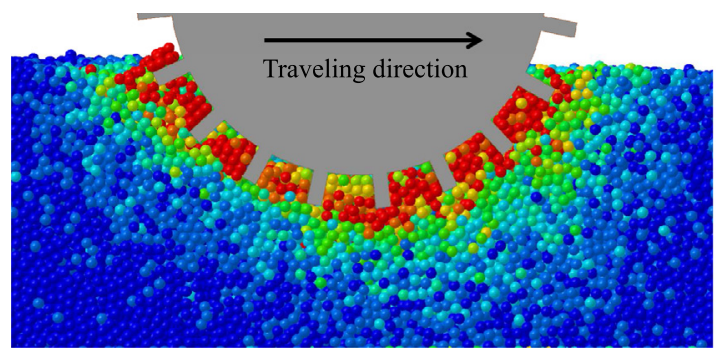

Fig. 14. Snapshots of the wheel-soil interaction in the DEM analysis, where Wheel B is used and the slippage is 0.5 . The colors in the contour map represent the magnitude of particle velocity, and the red part corresponds to the high velocity region. (For interpretation of the references to colour in this figure legend, the reader is referred to the web version of this article.)
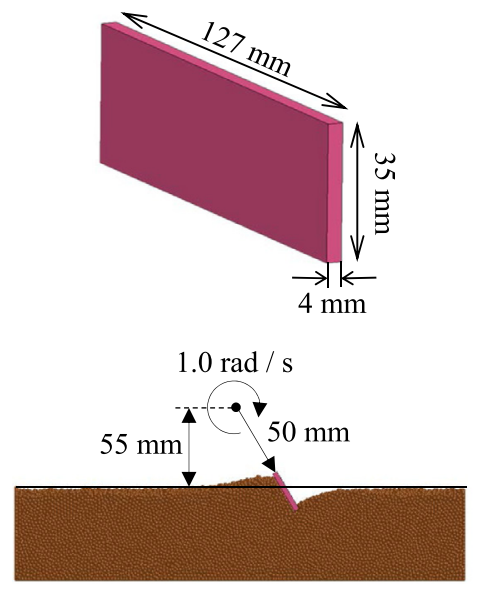

(a)

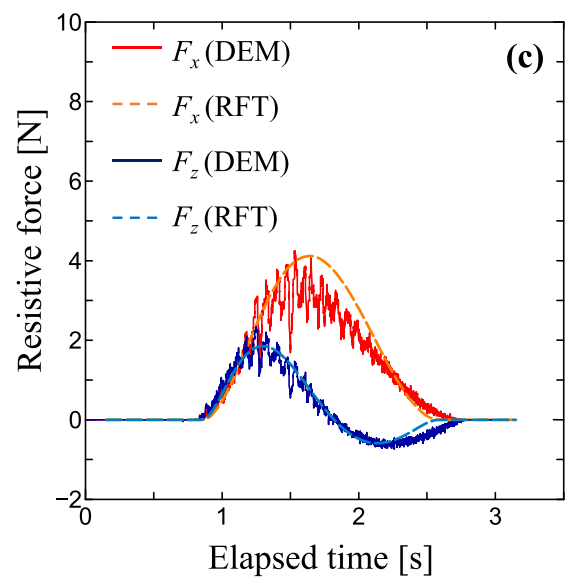

the plate in granular media, the moving behavior of soil toward the rear of wheel in a high-slippage regime is not considered. Therefore, as can be seen from Fig. 11, the relationship between sinkage and slippage in RFT is not properly evaluated. (Note that this shortcoming is the same for the conventional terramechanics model.)

The second reason is that, to fully demonstrate the resistive force, it is necessary for the plate to move independently. That is, when considering the movement of multiple plates, it is necessary that the distance between the plates is sufficiently secured, whereby the wheel with grousers examined in this study is not necessarily guaranteed this condition.

To examine the two above-mentioned reasons in more detail, DEM and RFT analyses of two types of plate test was carried out. Here, the soil condition is the same as in Fig. 3.

Fig. 15 shows a schematic diagram of the first analysis condition and obtained results. Fig. 15(a) shows a case where one independent plate rotates in the soil. On the other hand, the model, as shown in Fig. 15(b), consists of two plates, in which the second plate follows the first plate, moving in the same manner as in Fig. 15(a). Note that two plates maintain the parallel relationship and the distance between the normal lines with each other. Fig. 15(c) and (d) show the analysis results of variations of resistive forces in the horizontal direction and vertical direction obtained by DEM and RFT, respectively. The scale factor of RFT is obtained from Fig. 3 (i.e., $\zeta=0.191$ ). The solid line is the result of DEM, while the dashed line is the result of RFT. As can be seen from the figure, when one plate independently rotates in the soil-Fig. 15(a) and
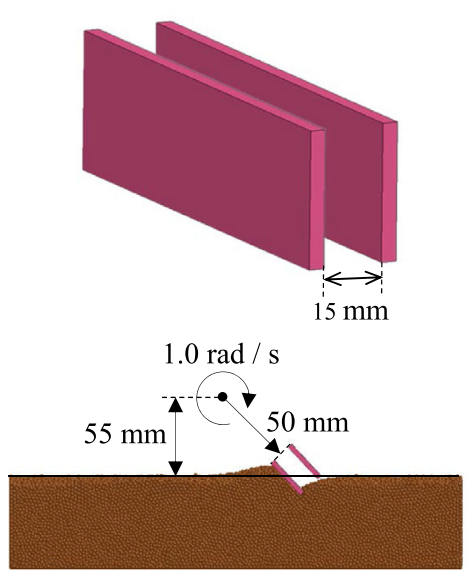

(b)

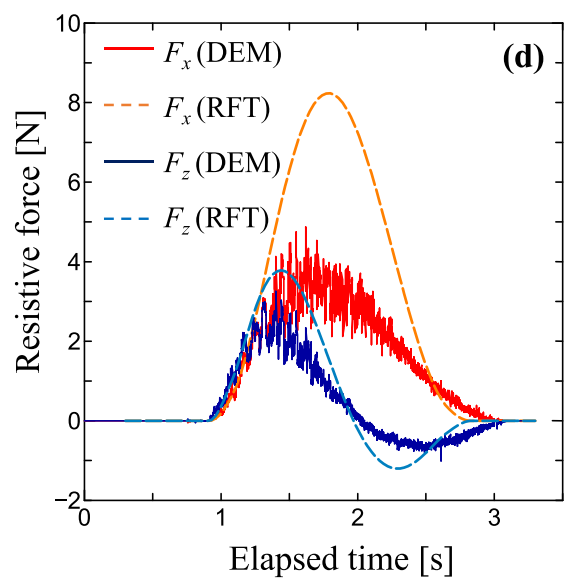

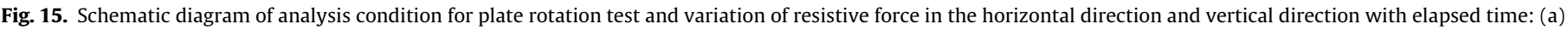
condition of single-plate rotation; (b) condition of two-plate rotation; (c) results of single-plate rotation; and (d) results of two-plate rotation. 

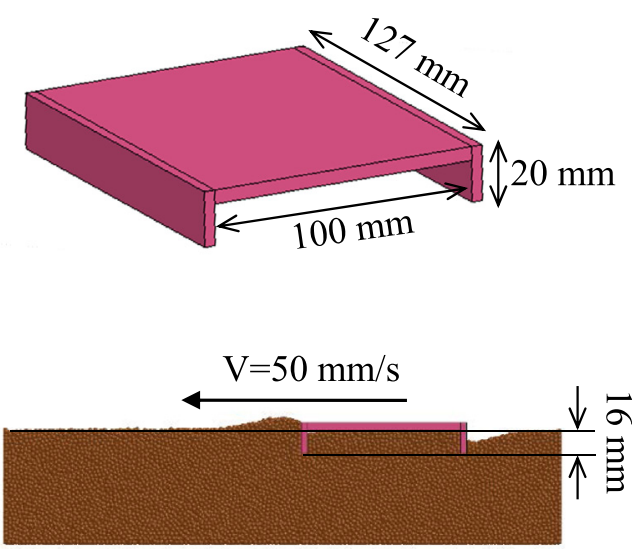

(a)

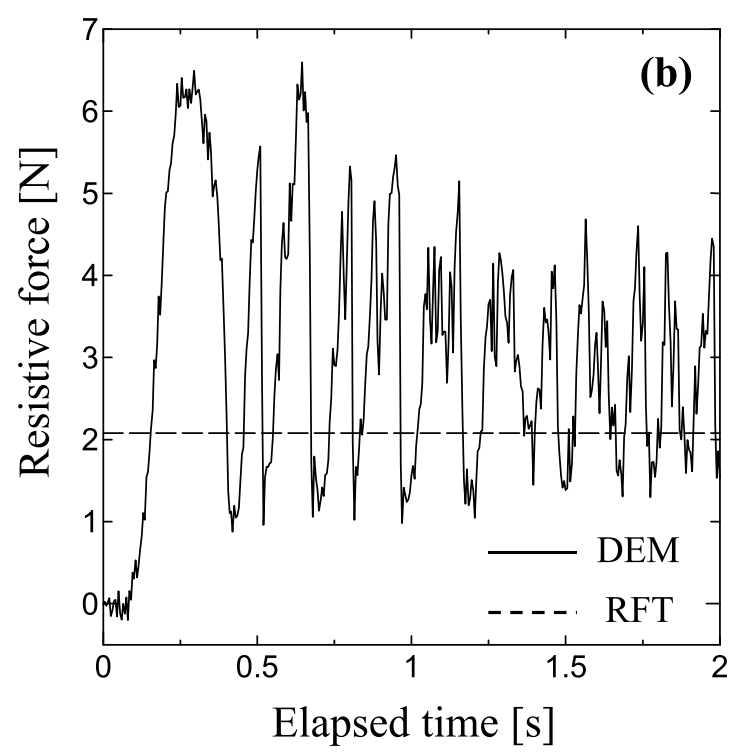

Fig. 16. Schematic diagram of analysis condition for bulldozing test of lidded plates and variation of resistive force in the horizontal direction with elapsed time: (a) condition of bulldozing test; (b) results of DEM and RFT analyses.

(c)-results obtained by DEM and RFT are in good agreement. However, in case of two plates-Fig. 15(b) and (d)-the result of DEM analysis is smaller than that of RFT. In RFT, if the plate has the same $\beta$ and $\gamma$, the same resistive force is generated. Hence, the force of the two-plate model is simply twice that of the one-plate model, based on the superposition principle. However, in DEM analysis, when the distance of the plate is short, the number of particles between plates decreases, and the resistive force generated in the rear plate is correspondingly reduced. When the distance between plates becomes sufficiently large, the analysis results obtained by DEM and RFT almost agree with each other, even in the case of multiple plates.

Fig. 16(a) shows a schematic diagram of the second analysis condition. In the analysis, a model with a lid attached to two plates penetrated soil by a fixed amount $(16 \mathrm{~mm})$, and then it moved at a constant velocity in the horizontal direction. Here, the two vertical plates have a distance such that they do not affect each other, and the sum of the resistive forces almost agrees with the analysis of RFT. Fig. 16(b) shows the variation of the resistive force obtained by DEM and RFT. The fluctuation of the result of DEM analysis occurs when getting over the particle. As can be seen from the figure, the value of the resistive force in the steady state is larger in DEM than the value calculated in RFT, because the packed soil particles between two vertical plates are subjected to vertical pressure by the lid and then generate additive driving force by forming a shear plane.

Based on the above two types of analysis results and the result of the particle velocity, shown in Fig. 14, when RFT is applied to the analysis of the wheel with grousers, the plate interval and shearing of particles cannot be ignored. RFT can predict the reaction force with sufficient accuracy for cases where the interaction between the object (plate) and granular media is dominant, such as a legged mobile robot. Meanwhile, to apply RFT to cases where the interaction between particles cannot be ignored, some extension of the model is necessary. Even so, we believe that the low calculation cost and high-precision performance of RFT is quite attractive, and we can use it for various terramechanics problems.

\section{Conclusion}

In this study, we examined the applicability of RFT for the analysis of a traveling wheel having a grouser by comparing it with the DEM analysis results. Specifically, we conducted plate intrusion/ extrusion and a wheel traveling analysis for a loose frictional soil as a virtual test based on the DEM. In plate tests, the results were revealed to be roughly consistent between DEM and RFT, but quantitative agreement was not confirmed in the wheel traveling analyses. To examine the discrepancy between DEM and RFT for the wheel traveling behavior, we conducted two additional analyses. We found that the discrepancy is because interaction between particles cannot be taken into consideration in RFT. In addition, we found that the distance between grousers also affects the resistive force. Based on the results and discussion, we believe that RFT might be a promising approach in terramechanics, although it is necessary to expand RFT to apply it to the analysis of the traveling behavior of a wheel with grousers. Note that RFT can easily be extended to three-dimensional problems. Therefore, RFT can also be applied to the problem of turning ability that is indispensable for multibody dynamics analysis of vehicles.

In this study, few kinds of numerical conditions were examined using the plane strain condition of DEM. Thus, the range of examined conditions is limited. To shows the robustness of RFT, various conditions should be considered. In addition, RFT should be verified by comparing it with a real test. We will address these issues in the future.

\section{Acknowledgments}

This work has been supported by the Impulsing Paradigm Change through Disruptive Technologies Program (ImPACT), Tough Robotics Challenge (TRC), JST, Japan.

\section{Appendix A. Supplementary material}

Supplementary data to this article can be found online at https://doi.org/10.1016/j.jterra.2019.01.001.

\section{References}

Arvidson, R., Iagnemma, K., Maimone, M., Fraeman, A., Zhou, F., Heverly, M., Belutta, P., Rubin, D., Stein, N., Grotzinger, J., Vasavada, A., 2017. Mars science laboratory curiosity rover megaripple crossings up to Sol 710 in gale crater. J. Field Rob. 34 (3), 495-518.

Askari, H., Kamrin, K., 2016. Intrusion rheology in grains and other flowable materials. Nat. Mater. 15, 1274-1279.

Chiroux, R.C., Foster Jr., W.A., Johnson, C.E., Shoop, S.A., Raper, R.L., 2005. Threedimensional finite element analysis of soil interaction with a rigid wheel. Appl. Math. Comput. 162, 707-722.

Fervers, C.W., 2004. Improved FEM simulation model for tire-soil interaction. J. Terramech. 41 (2-3), 87-100.

Hambleton, J.P., Drescher, A., 2008. Modeling wheel-induced rutting in soils: indentation. J. Terramech. 45 (6), 201-211. 
Hambleton, J.P., Drescher, A., 2009. Modeling wheel-induced rutting in soils: rolling. J. Terramech. 46 (2), 35-47.

Heiken, G., Vaniman, D., French, B., 1991. Lunar Soucebook.

Ishigami, G., Miwa, A., Nagatani, K., Yoshida, K., 2007. Terramechanics-based model for steering maneuver of planetary exploration rovers on loose soil. J. Field Rob. 24 (3), 233-250.

Jiang, M., Liu, F., Shen, Z., Zheng, M., 2014. Distinct element simulation of lugged wheel performance under extraterrestrial environmental effects. Acta Astronaut. 99, 37-51.

Johnson, J., Kulchitsky, A., Duvoy, P., Iagnemma, K., Senatore, C., Arvidson, R., Moore, J., 2015. Discrete element method simulations of Mars Exploration Rover wheel performance. J. Terramech. 62, 31-40.

Johnson, L.L., King, R.H., 2010. Measurement of force to excavate extraterrestrial regolith with a small bucket-wheel device. J. Terramech. 47 (2), 87-95.

Knuth, M.A., Johnson, J.B., Hopkins, M.A., Sullivan, R.J., Moore, J.M., 2012. Discrete element modeling of a Mars Exploration Rover wheel in granular material. J. Terramech. 49 (1), 27-36.

Kobayashi, T., Ochiai, H., Suyama, Y., Aoki, S., Yasuhuku, N., Omine, K., 2009. Bearing capacity of shallow foundations in a low gravity environment. Soils Found. 49 (1), 115-134.

Li, C., Zhang, T., Goldman, D.I., 2013. A terradynamics of legged locomotion on granular media. Science 339 (6126), 1408-1412.

Li, W., Huang, Y., Cui, Y., Dong, S., Wang, J., 2010. Trafficability analysis of lunar mare terrain by means of the discrete element method for wheeled rover locomotion. J. Terramech. 47 (3), 161-172.

LSCT, LS-DYNA User's Manual, 2017. LSCT Singapore.

Nakashima, H., Fujii, H., Oida, A., Momozu, M., Kawase, Y., Kanamori, H., Aoki, S., Yokoyama, T., 2007. Parametric analysis of lugged wheel performance for a lunar microrover by means of DEM. J. Terramech. 44 (2), 153-162.

Nakashima, H., Fujii, H., Oida, A., Momozu, M., Kanamori, H., Aoki, S., Yokoyama, T., Shimizu, H., Miyasaka, J., Ohdoi, K., 2010. Discrete element method analysis of single wheel performance for a small lunar rover on sloped terrain. J. Terramech. 47 (5), 307-321.

Nishiyama, K., Nakashima, H., Yoshida, T., Ono, T., Shimizu, H., Miyasaka, J., Ohdoi, K., 2016. 2D FE-DEM analysis of tractive performance of an elastic wheel for planetary rovers. J. Terramech. 64, 23-35.
Ozaki, S., Hinata, K., Senatore, C., Iagnemma, K., 2015. Finite element analysis of periodic ripple formation under rigid wheels. J. Terramech. 61, 11-22.

Ozaki, S., Kondo, W., 2016. Finite element analysis of tire traveling performance using anisotropic frictional interaction model. J. Terramech. 64, 1-9.

Shikanai, T. Hashiguchi, K. Nohse, Y. Ueno, M. Okayasu, T, 2000. Precise measurement of soil deformation and fluctuation in drawbar pull for steel and rubber-coated rigid wheels. J. Terramech. 37 (1), 21-39.

Slonaker, J., Motley, D.C., Zhang, Q., Townsend, S., Senatore, C., Iagnemma, K. Kamrin, K., 2017. General scaling relations for locomotion in granular media. Phys. Rev. E 95, 052901.

Smith, W., Peng, H., 2013. Modeling of wheel-soil interaction over rough terrain using the discrete element method. J. Terramech. 50 (5-6), 277-287.

Sutoh, M., Nagatani, K., Yoshida, K., 2011. Evaluation of influence of surface shape of wheel on traveling performance of planetary rovers over slope. Proceedings of the 17th International Conference of the ISTVS - Virginia, USA, 18-22 November 2011. CD-Publication.

Sutoh, M., Yusa, J., Ito, T., Nagatani, K., Yoshida, K., 2012. Traveling performance evaluation of planetary rovers on loose soil. J. Field Rob. 29 (4), 648-662.

Suzuki, H., Ozaki, S., 2017. Terramechanics analysis of wheel with grousers based on resistive force theory. Proceedings of the 19th International and 14th EuropeanAfrican Regional Conference of the ISTVS - Budapest, Hungary, 25-27 September 2017. CD-Publication (Paper 105).

Suzuki, H., Ozaki, S., Otsuki, M., Senatore, C., Iagnemma, K., 2016. RFT-based analysis of vehicle-terrain interaction problems. Proceedings of the 8th Americas Regional Conference of the ISTVS - Detroit, USA, 12-14 September 2016. CDPublication (Paper 59).

Wong, J.Y., 2012. Predicting the performances of rigid rover wheels on extraterrestrial surfaces based on test results obtained on earth. J. Terramech. 49 (1), 49-61.

Xia, K., 2011. Finite element modeling of tire/terrain interaction: application to predicting soil compaction and tire mobility. J. Terramech. 48 (2), 113-123.

Zhang, T., Goldman, D.I., 2014. The effectiveness of resistive force theory in granular locomotion. Phys. Fluids 26, 101308.

Zhou, F., Arvidson, E., Bennett, K., Trease, B., Lindemannm, R., Bellutta, P., Iagnemma K., Senatore, C., 2014. Simulations of mars rover traverses. J. Field Rob. 31 (1), 141-160. 